\title{
Ultrasonic phased array inspection of a wire plus arc additive manufactured (WAAM) sample with intentionally embedded defects
}

\author{
Yashar Javadi $^{1 *}$, Charles N. MacLeod ${ }^{1}$, Stephen G. Pierce ${ }^{1}$, Anthony Gachagan ${ }^{1}$, David \\ Lines $^{1}$, Carmelo Mineo ${ }^{1}$, Jialuo Ding ${ }^{2}$, Stewart Williams ${ }^{2}$, Momchil Vasilev ${ }^{1}$, Ehsan \\ Mohseni ${ }^{1}$ and Riliang $\mathrm{Su}^{1}$ \\ ${ }^{1}$ Centre for Ultrasonic Engineering (CUE), Department of Electronic \& Electrical \\ Engineering, University of Strathclyde, Glasgow G1 1XQ, UK \\ ${ }^{2}$ Welding Engineering and Laser Processing Centre, School of Aerospace, Transport and \\ Manufacturing Building 46, Cranfield University, Cranfield, Bedfordshire MK43 OAL, UK \\ *Corresponding author's email: yashar.javadi@strath.ac.uk
}

\begin{abstract}
In this study, Wire + Arc Additive Manufacture (WAAM) was employed to manufacture a steel specimen with intentionally embedded defects which were subsequently used for calibration of an ultrasonic phased array system and defect sizing. An ABB robot was combined with the Cold Metal Transfer (CMT) Gas Metal Arc (GMA) process to deposit 20 layers of mild steel. Tungsten-carbide balls (ø1-3 mm) were intentionally embedded inside the additive structure after the $4^{\text {th }}, 8^{\text {th }}, 12^{\text {th }}$ and $18^{\text {th }}$ layers to serve as ultrasonic reflectors, simulating defects within the WAAM sample. An ultrasonic phased array system, consisting of a $5 \mathrm{MHz} 64$ Element phased array transducer, was used to inspect the WAAM sample nondestructively. The majority of the reflectors were detected successfully using Total Focusing Method (TFM), proving that the tungsten carbide balls were successfully embedded during the WAAM process and also that these are good ultrasonic reflectors. Owing to a lack of standards and codes for the ultrasonic inspection of WAAM samples (A. Lopez, R. Bacelar, et al., 2018), a calibration method and step-by-step inspection strategy were introduced and then used to estimate the size and shape of an unknown lack of fusion (LoF) indication. This was then validated by destructive analysis, showing a good correlation with the phased array results.
\end{abstract}

Keywords: Wire + Arc Additive Manufacture (WAAM); Ultrasonic Phased Array; Total Focusing Method (TFM); Intentionally Embedded Defects. 


\section{Introduction}

Building 3D samples by adding layer-upon-layer of material is known as Additive Manufacturing (AM), which is seeing increasing interest and use in the field of manufacturing engineering. This is driven by technical and commercial advantages in the manufacture of complex parts through more cost-effective approaches. AM can produce less material wastage, shorter time to market, more design freedom, reduction of overall part weight and reduced complexity in comparison with the assembly and joining of many subcomponents typically deployed in other manufacturing processes [1]. However, since no single AM technique can bring all the advantages together simultaneously, it is critical to choose an AM method accurately, based on the application. The working envelope of laser and electron beam Powder Bed Fusion (PBF) limits the maximum component size [2], however, the recent industrial developments allow the production of parts up to $900 \mathrm{~mm}$ wide through using large PBF 3D printers [3]. The component size is particularly important for the aerospace industry where the demand is for ever larger and more complex structures with lower Buy-To-Fly (BTF) ratio [1]. The necessity of reaching a lower BTF is justified by the increasing use of expensive titanium rather than aluminium (due to its electrochemical incompatibility with carbon fibre) [4]. Currently, large aircraft components (e.g., stiffened panels, wing ribs, etc.) are manufactured by machining from billets or large forgings and where a BTF ratio of 10 or even 20 is not unusual [5]. Wire + Arc Additive Manufacture (WAAM) has been successfully employed for the purpose of BTF reduction in large components [5]. For example, Williams et al [5] highlighted material savings in excess of roughly $500 \mathrm{~kg}$ in a $2.5 \mathrm{~m}$ aluminium wing rib due to a BTF reduction from 37 (traditional manufacturing methods) to 12 by WAAM [5]. Although there is a capability of producing 10 $\mathrm{m}$ long WAAM components [6], a 6-meter long aluminium WAAM part is believed to be one of the largest metal 3D parts ever made [7]. Regarding the deposition rate, a review on the AM methods by Ding et al [8] showed that WAAM deposition rate can reach to $12 \mathrm{~g} / \mathrm{min}$ which is higher than most of the powder-based AM methods (e.g., selective laser sintering delivers just $0.1 \mathrm{~g} / \mathrm{min}$ ). Furthermore, WAAM has a lower capital cost in comparison with the laser and powder-based technologies, while no powder handling is required and finally a higher material usage efficiency can be achieved [9].

Every safety-critical component, including any AM part, needs to be inspected after manufacturing to ensure absence of any concerning defects, no material degradation or deviation from the designed characteristics of the component. Non-destructive testing (NDT) 
methods allow assessing manufactured parts and evaluating the properties of their material, without causing any damage. NDT methods can be carried out by skilled personnel, or emerging automated approaches. They provide quantitative information of the asset condition, which is used to evaluate the remaining lifetime of the asset and help to decide when replacement or decommissioning is required. This maximises the asset usage, whilst guaranteeing safety. Automated NDT approaches are increasingly been used for assets requiring inspection in the areas difficult to access and hazardous to human beings $[10,11]$. This along with the increased demand for higher inspection accuracy and efficiency has underpinned a research and development drive to automate current NDT inspection techniques [10-12], in order to improve accuracy by reducing the weakest link in the NDT supply chain, i.e., human error [13].

As high-technology electronics are continuously delivered to the market with lower cost, it is increasingly common to use a linear array of ultrasonic transducers, each with its own sending and receiving electronics, rather than a single element probe [14]. In comparison with single element transducers, ultrasonic arrays provide wider scanning areas, focusing capability, a higher inspection quality with flexibility and less inspection time due to an ability to produce a rapid visualisation. Moreover, phased array probes allow the undertaking of a range of different inspections from a single location by synthetic aperture focusing and the possibility to steer the ultrasonic beam over a range of angles and positions [15-17]. The development of 2D phased-array ultrasonic imaging transducers could also increase the application of 3D volumetric imaging of components [18]. Furthermore, high-temperature arrays [19], flexible arrays [20] and air-coupled arrays [21, 22] are increasingly attractive for NDT of the components in harsh environments, particularly nuclear and aerospace industries.

As WAAM components are increasingly being considered for safety-critical industries such as aerospace, the rapid development of NDT methods and structural integrity assessment of WAAM specimens is essential. However, there is no standard and code for the NDT of AM parts, including ultrasonic inspection of WAAM samples [23]. The importantance of calibration procedure is reflected in the joint collaboration between International Organization for Standardization (ISO) and ASTM in which NDT for AM parts, ISO/TC 261/JG 59, is due to be published by end of 2019 [24]. NDT for AM parts was reviewed by Clark et al [25], who highlighted the need to develop a more advanced system for accurate detection of porosities and lack-of-fusion defects between the AM layers. Lopez et al [23, 26] reviewed the application of various NDT techniques, including radiography and ultrasound, 
and evaluated the defect detection capability of these methods on the WAAM samples. However, they employed single element ultrasonic probes, rather than the ultrasonic phased array transducers considered in this paper, and surface finish was highlighted as a scanning issue in the WAAM samples [23]. Ultrasonic phased array inspection of a WAAM sample was discussed by Javadi et al [27] for aluminium parts.

Specimens with intentionally embedded deposition defects or flaws can be employed for NDT training, and for researching and developing new NDT procedures targeted to AM components [28]. Such specimens are very valuable, since they contain realistic and representative volumetric deposition defects. For example, tungsten rods embedded between the deposited layers have been used to represent lack of fusion more realistically than a side-drilled hole $(\mathrm{SDH})$ in the deposit $[28,29]$. Furthermore, the process of the intentional deposition defect is mainly independent from the material machinability. This is beneficial for the materials which are hard to machine (e.g., Titanium) and becomes a suitable alternative for machining of small SDH. Tungsten carbide balls, as an example of intentional deposition defects [29], are known size defects (ø1-3 mm in this study) which can be used for calibration and sizing of the unknown size defect indications. Tungsten carbide balls, embedded in the weld, were successfully detected by Javadi et al [29], however, no accurate sizing techniques of such intentional defects have been investigated to date. In this paper, tungsten carbide balls are embedded in a WAAM wall to provide a framework for designing a calibration procedure. Furthermore, the ultrasonic phased array setup and Total Focusing Method (TFM) post-processing approach are used. This innovative combination of aspects is then used not only for detection of an unknown size defect but also for sizing and shape-estimation of that defect. Defect sizing is not possible without an application of a proper calibration procedure, which is introduced in this paper through the use of intentionally-embedded tungsten in the WAAM sample. This calibration procedure is proposed to enhance the inspection accuracy of the WAAM samples in the absence of any standards and codes for NDT of AM parts.

\section{Theoretical background}

A WAAM sample is a 3D printed specimen which is built by adding layer-upon-layer of material on a base plate using an electric arc to melt and deposit the wire, see Figure 1a. The bottom surface inspection process of the WAAM sample is schematically shown in Figure 
1b. This approach was used for inspecting aluminium WAAM samples by Javadi et al [27], however, top surface inspection (see Figure 1c) has a higher potential to be used for the inprocess WAAM inspection. The WAAM top surface is machined to facilitate an application of a flat-surface wedge. The probe is equipped with a standoff wedge to avoid missing nearsurface reflectors due to the ultrasonic near field of the probe (Figure 1c). Assuming the presence of four artificial reflectors in the WAAM sample (Figure 1b-c) three main approaches can be used to detect these reflectors: plane B-scan, focused B-scan and Total Focusing Method (TFM), respectively represented in Figure 1d-f.

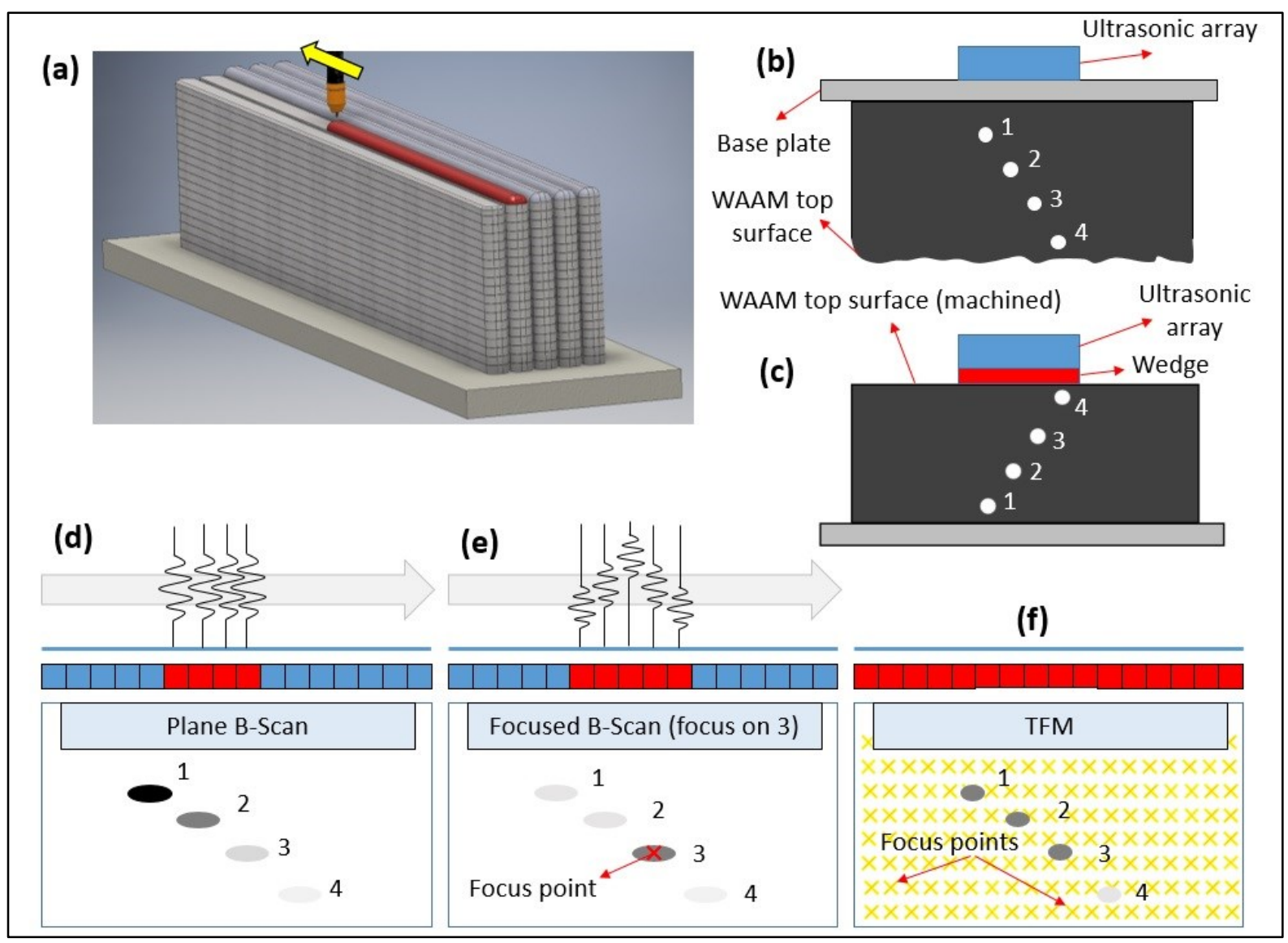

Figure 1. WAAM manufacturing process (a) and phased array inspection (b: bottom surface inspection and c: top surface inspection) using plane B-scan (d), focused B-scan (e) and TFM (f)

A B-scan (see Figure 1d) is produced using a planar front ultrasonic beam, generated by a group of adjacent elements (aperture) which are simultaneously pulsed. A single signal is then produced by summing all the echo signals (A-scans) received from the aperture elements. The performance is equivalent to a single element unfocused transducer as large as the aperture. The final B-scan image is then produced by moving the aperture electronically 
along the array length. Focusing can improve both sensitivity and rendition of the defect topology at the focal point. However, the beam divergence beyond the focus is increased and so a compromise value of aperture and focus is usually chosen so as to match the depth of field for the application requirements [14].

Although the focusing can help to show the defect shape more accurately, regardless of its position, it does not remove the challenges related to the penetration. Ultrasound waves are attenuated when they penetrate through the thickness of the specimen. As the WAAM specimen is a multi-layer deposition, penetrating deeper can be more challenging for the ultrasonic beam, which is required to travel through a larger number of anisotropic layers produced by multiple layers. Then, the signal-to-noise ratio reduces with the inspection depth. Therefore, a number of WAAM defects, which are positioned far from the scanning surface, can be missed. That is why the Focused B-scan (see Figure 1e) is advantageous for scanning of the deep WAAM defects. If differential time delays are introduced for the elements within an aperture at both the transmission and reception stages, a focused beam is produced [17]. However, manually setting the correct pulse delays to focus the ultrasonic energy on many points and cover the full volume of interest is time-consuming. Therefore, this paper considers the Full Matrix Capture (FMC) and Total Focusing Method (TFM) to ensure focusing for all points within the produced image (see Figure 1f). FMC involves the collection of A-scans from all elements of the array, corresponding to successive transmissions of each element, firing one at a time. This approach is used to maximise the information extracted from an array $[16,17]$. TFM is an imaging algorithm that uses data acquired in FMC mode for post-processing and then all elements in the array are employed to focus at every single point in the image. The number of focal depths in transmission and reception is unlimited [17] in TFM scanning of the WAAM sample. TFM can be implemented after capturing the FMC data, off-line, or during the scanning, real-time TFM, while the latter approach requires a dedicated post-processing software as well as high computational power computer.

\section{Manufacturing process (WAAM)}

Figure 2 shows the manufacturing setup and the facilities for embedding intentional-defects in the WAAM sample. The base plate was $12 \mathrm{~mm}$ thick and made of ground-to-bright-metal mild steel (EN 10025 S275); it was clamped in six points, as shown in Figure 2. A Gas Metal 
Arc (GMA) torch mounted on a six-axis ABB robot along with a cold metal transfer (CMT) power source was used. The wire was $\varnothing 1.2 \mathrm{~mm}$ SupraMig Ultra (AWS A5.18 ER70S-6) and the shielding gas was Argoshield Light (O2 2\%; CO2 $5 \%$; Ar $93 \%$ - Welding mixture ISO 14175-M14-ArCO-5/2). In total, 20 layers were deposited with 5 passes in each layer. Travel speed was set in the robot program at a fixed value of $6.67 \mathrm{~mm} / \mathrm{s}$. The wire feeding speed (WFS) was set on the power source, with the possibility of adjusting it during manufacturing, without pausing the WAAM process. The WFS was $5 \mathrm{~m} / \mathrm{min}$ for the $1^{\text {st }}$ layer of WAAM, 4.5 $\mathrm{m} / \mathrm{min}$ for the $2^{\text {nd }}, 3^{\text {rd }}$ and $4^{\text {th }}$ layers but $4 \mathrm{~m} / \mathrm{min}$ for all other layers. The increase in WFS of the first four layers avoids deposition defects by giving a higher heat input for the initial layers where the substrate is close.

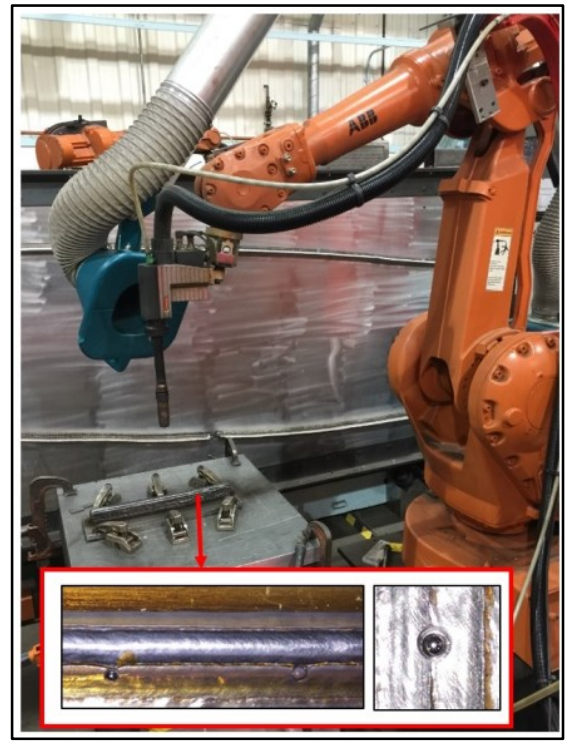

Figure 2. Manufacturing setup for the WAAM sample with the intentionally embedded tungsten carbide balls

The tungsten balls, placed inside the holes produced on the WAAM surface using a portable drilling machine, were embedded after Layer 4, 8, 12 and 18 (Figure 3). These tungsten balls will be later used to design a calibration procedure and step-by-step inspection strategy by which an unknown defect (with any morphology) can be sized. These are not, therefore, representing the exact morphology expected from the WAAM defects, which are mainly expected to be lack of fusion for the sample investigated in this work [5]. Alternatively, the combination of tungsten and air-filled gaps around them will serve as the known size defects which are used to calibrate the ultrasonic inspection system. It is worth mentioning that a combination of tungsten balls and rods similar to those used in welds by Javadi et al [29] can 
be a more realistic representation of defects. However, tungsten rods were not considered in this work, due to some practical difficulties of using them in the WAAM wall. Inspection of porosity is also not considered in this paper, as it will need a higher sensitivity inspection system. It should be noted that the minimum defect size detectable with the new calibration procedure developed in this paper, is limited to $>0.5 \mathrm{~mm}$ (will be discussed later in Figure 10) which is not small enough to detect the WAAM porosity [30, 31]. Furthermore, it is believed that the likelihood of generating porosities in the WAAM sample was minimised by using optimum manufacturing parameters in this work $[5,30,31]$. Therefore, although this is not the case for all WAAM components, porosity is not a manufacturing concern for the samples investigated in this paper.

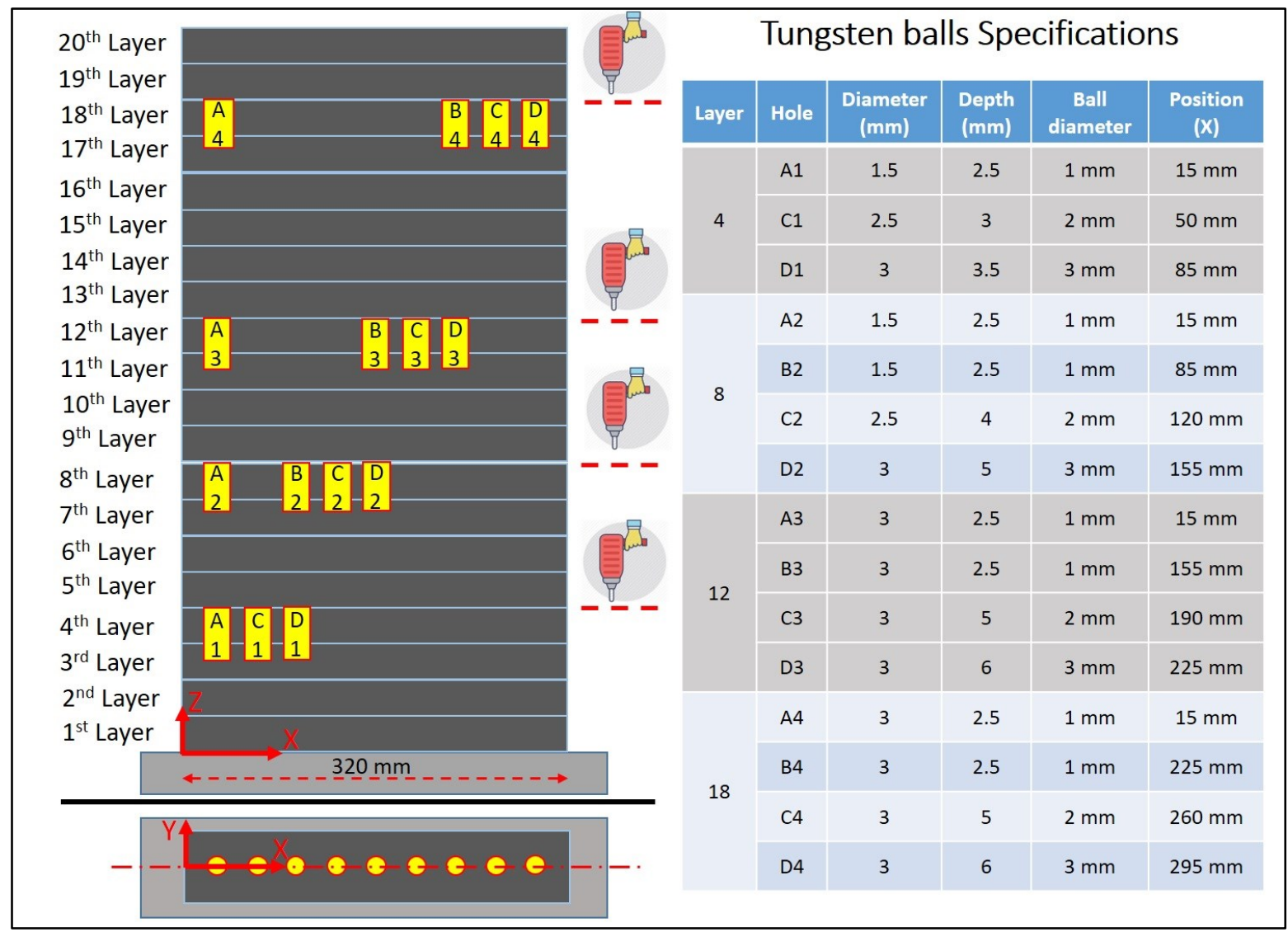

Figure 3. Specifications and position of the tungsten balls placement inside the WAAM sample

\section{Inspection results and discussions}

The ultrasonic phased array setup comprised the FIToolbox controller (Diagnostic Sonar, UK) and a $5 \mathrm{MHz}$ Olympus array (64 elements $0.5 \mathrm{~mm}$ pitch) mounted on a 20mm zerodegree Rexolite wedge (Olympus). There is no existing standard or code available for the 
phased array inspection of WAAM samples. Therefore, as part of the work presented here, a calibration procedure has been designed. In this study, a standard phased array calibration block was initially used (see Figure 4a). Because the material and dimensions of the standard block are different from the WAAM specimen, it was decided to manufacture a calibration sample with the same WAAM wall height and the base plate material which was S275 (see Figure $4 b)$. However, since the S275 calibration sample does not have exactly the same acoustic properties of the WAAM sample, a number of SDHs were made in the WAAM specimen too (see Figure 4c). Some of the holes $(\varnothing 1.5 \mathrm{~mm}$ and $\varnothing 2 \mathrm{~mm})$ are not through-holes in order to show the necessity of performing the meander scan (Figure 4d), which is discussed later. The calibration process for the $ø 3 \mathrm{~mm} \mathrm{SDH}$ is shown in Figure 5. All the images are normalised to $80 \%$ of the maximum reflection amplitude received from the $\varnothing 3$ $\mathrm{mm}$ SDH in the S275 reference block. Therefore, any reflector with an amplitude higher than this amount appears black in colour as shown in the dB scale bar (see Figure 5). The S275 reference sample required a gain of $41 \mathrm{~dB}$ to bring the indication relative to the $ø 3 \mathrm{~mm} \mathrm{SDH}$ to the black colour threshold. Although higher attenuation is expected in the WAAM sample (lower gain required in comparison with the S275 reference sample), $35 \mathrm{~dB}$ gain was sufficient for WAAM specimen. This unexpected finding, due to the behaviour of the ultrasonic wave propagation inside the WAAM sample, was the main justification behind the drilling of SDHs for calibration purpose (Figure 4c). Furthermore, due to the inhomogeneous microstructure of the WAAM sample through its thickness, which includes five passes, the meander scan produces slightly different results at every scanning pass. Indeed, the gain dropped from $35 \mathrm{~dB}$ to $32 \mathrm{~dB}$ by moving the probe position in the y-axis direction, when scanning the through-hole reflector (see Figure 5). 


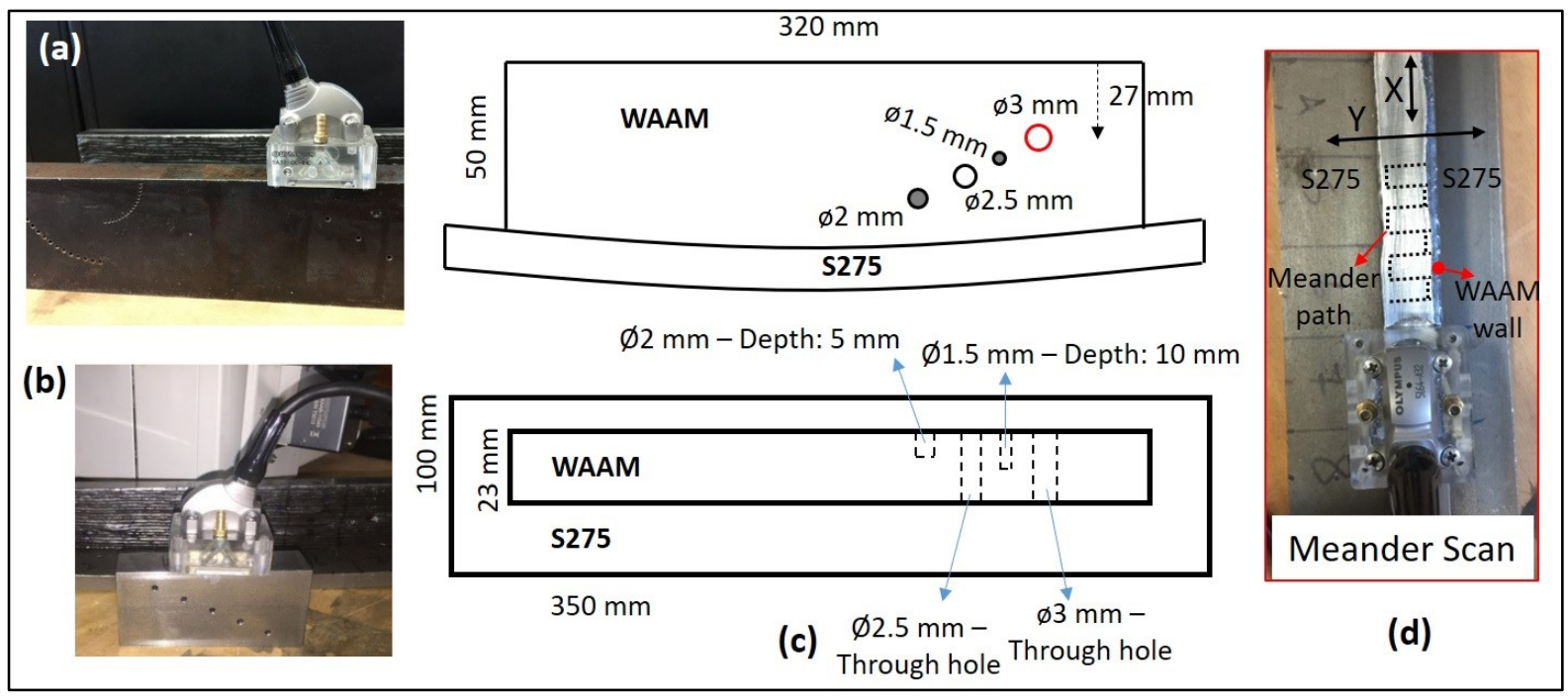

Figure 4. Ultrasonic calibration for inspection of the WAAM sample using (a) standard phased array calibration block, (b) S275 calibration sample with the same height of the WAAM sample, (c) known size SDH on the WAAM sample and (d) direction of Meander Scan

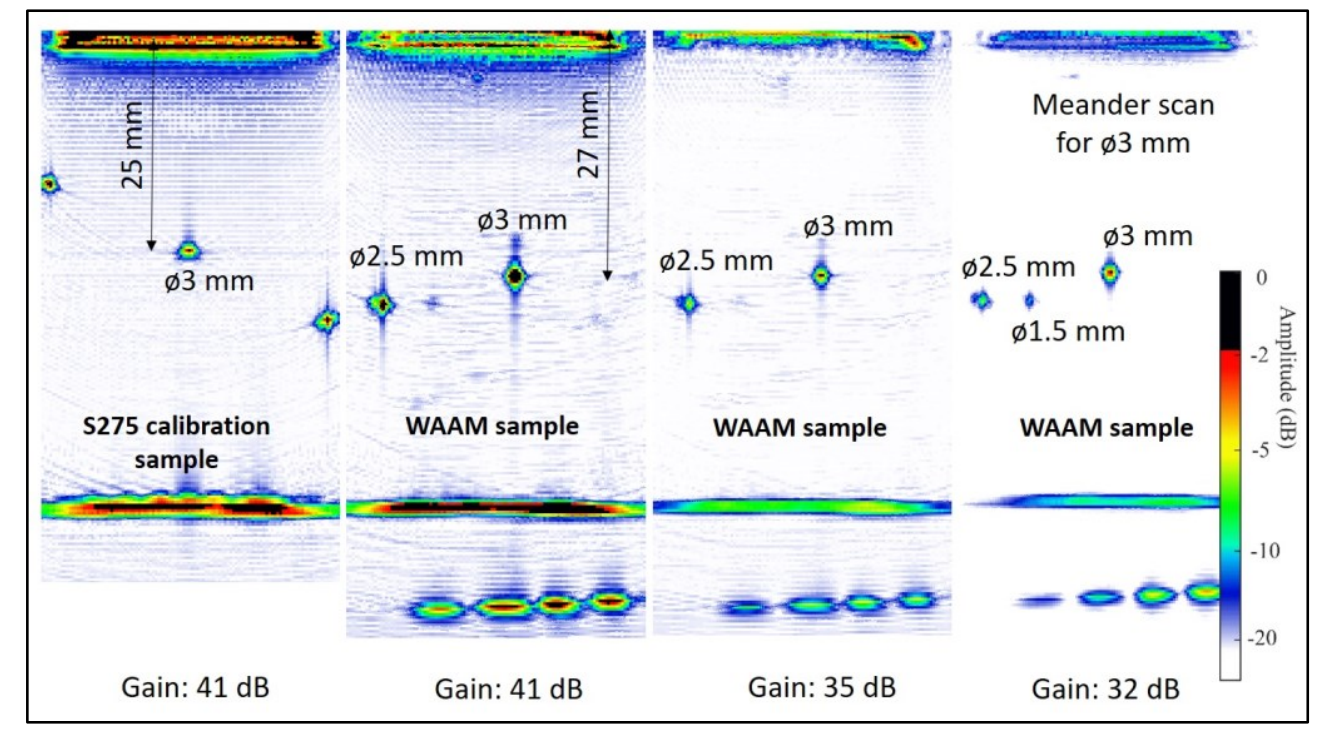

Figure 5. Calibration of $ø 3 \mathrm{~mm}$ SDH

Similarly, the meander scanning of the other SDHs on the WAAM sample was carried out and the results are summarised in Figure 6. For example, keeping the gain equal to $41 \mathrm{~dB}$, if an indication breaks the black colour threshold on the UT image, it can be concluded that such defect has size equal or greater than the $ø 1.5 \mathrm{~mm} \mathrm{SDH}$. Then, in order to cover the full length of the whole WAAM sample, 11 consecutive sections were scanned using real-time 
TFM (under constant $41 \mathrm{~dB}$ gain). Given the array length is $30 \mathrm{~mm}, 11$ sections guaranteed that the whole $320 \mathrm{~mm}$ WAAM wall length was swept in the $\mathrm{x}$-axis direction (Figure 7).

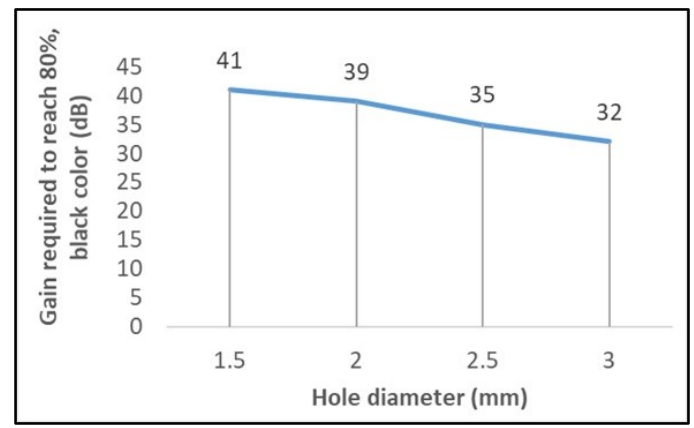

Figure 6. Calibration results: gain required to detect the SDH on the WAAM sample

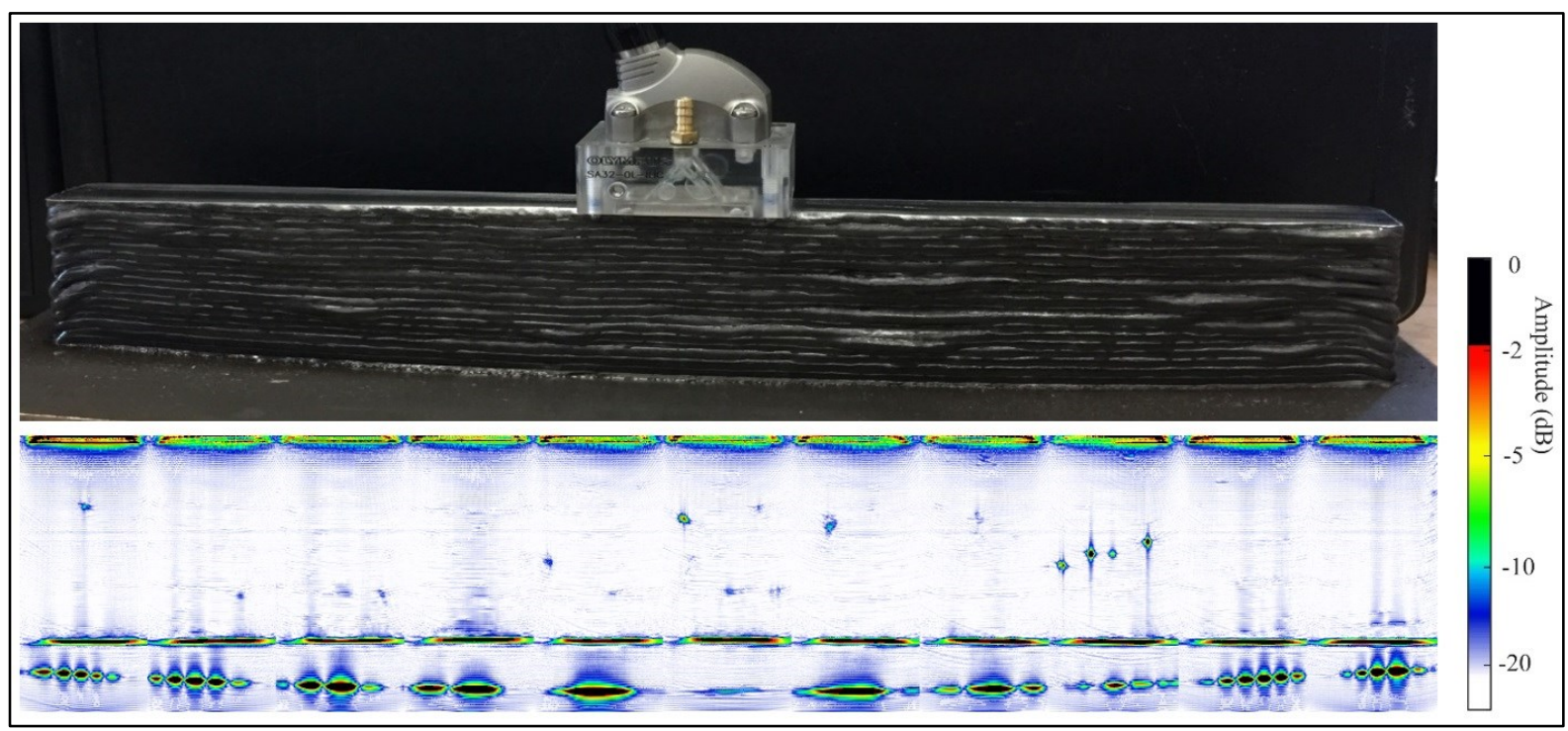

Figure 7. TFM image of the WAAM sample

The TFM images show a number of reflectors relative to the position of tungsten carbide balls. However, the TFM images are captured by discretely moving the probe along the $\mathrm{X}$ axis direction, while keeping the centre of the wedge at the centre of the WAAM sample top surface for all 11 sections (no scanning in the y-axis direction). All tungsten balls should have been positioned in the WAAM wall centre during manufacturing. However, small positional deviations between the final placement of the tungsten balls and the middle wall section cannot be ruled out, due to both hole drilling and ball placement being manually performed. Meander scanning was carried out in correspondence of each reflector, in order to monitor 
the ultrasonic response in the y-axis direction (Figure 8a). The results relative to the SDHs machined in the WAAM sample and ultrasonically detected using the same gain $(41 \mathrm{~dB})$ are shown in Figure 8b. The gain required to reach the $80 \%$ amplitude (black colour threshold) for $ø 3 \mathrm{~mm}$ ball (D1, D2 and D3) is $53 \mathrm{~dB}(+12 \mathrm{~dB}$ in comparison with the reference $41 \mathrm{~dB})$ as shown in Figure 8c. The required gain increment is within $7 \mathrm{~dB}$ and $14 \mathrm{~dB}$ for $\varnothing 2 \mathrm{~mm}$ and $\varnothing 1 \mathrm{~mm}$ balls, respectively (see Figure $8 \mathrm{~d}, \mathrm{e}$ ). It is worth mentioning that there is a deviation between the hole diameter and the ball diameter; for example, $\mathrm{C} 3$ was a $\varnothing 2 \mathrm{~mm}$ ball placed in a $ø 3 \mathrm{~mm}$ hole (see Figure 3).

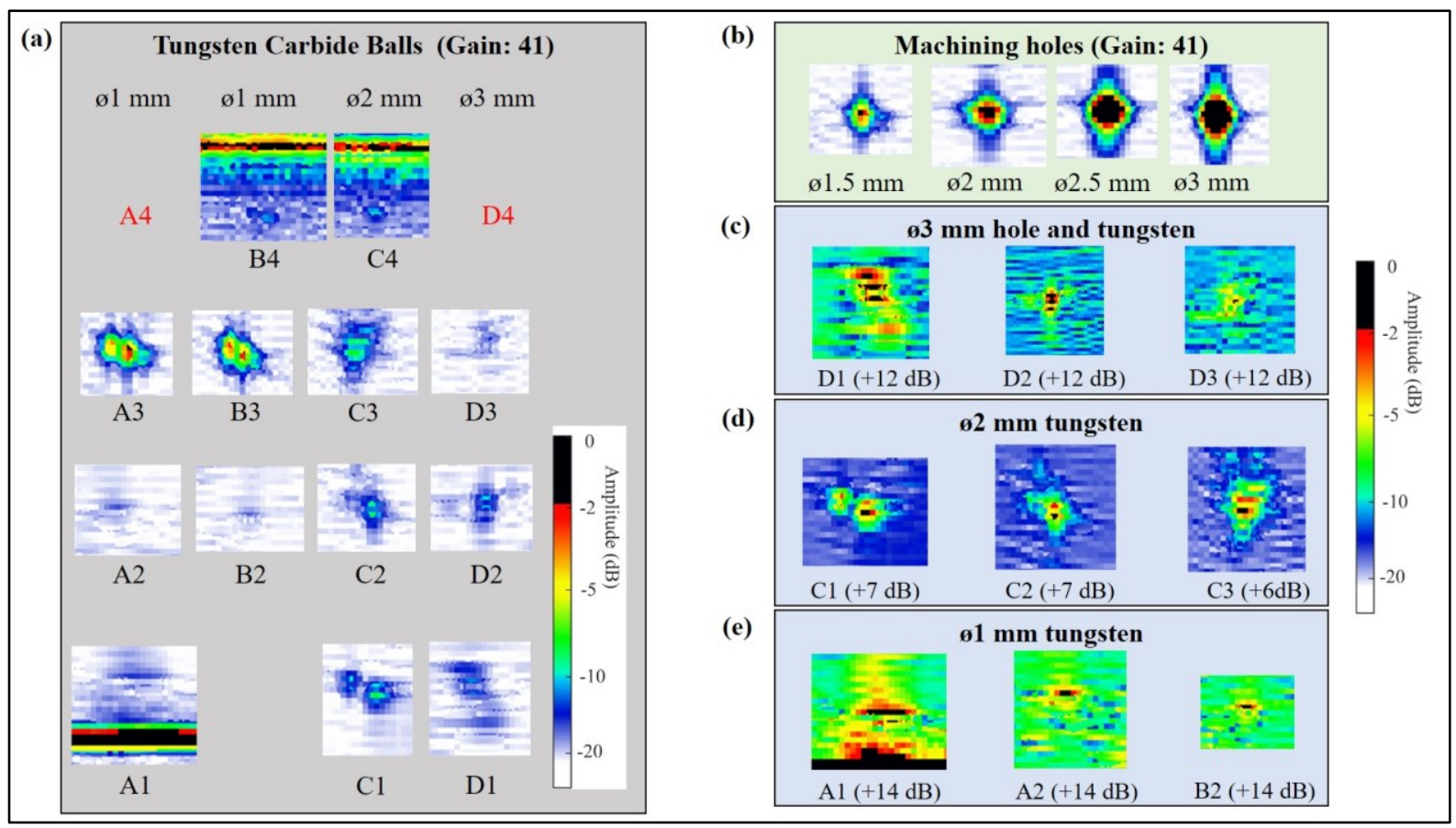

Figure 8. TFM and meander scanning results of all tungsten carbide balls (a) and SDH machined on the WAAM sample (b). The gain increase (in comparison with the reference $41 \mathrm{~dB}$ ) required to capture black colour for (c) $\varnothing 3 \mathrm{~mm}$, (d) $\varnothing 2 \mathrm{~mm}$ and (e) $\varnothing 1 \mathrm{~mm}$ tungsten balls.

The discrepancy between hole diameter and ball diameter, plus their inaccurate positions are the reasons behind a number of assumptions (Figure 9), which support the interpretation of the results (see summary charts as shown in Figure 10) and the formulation of the conclusions listed below (the numbering refers to Figure 11):

1) A3 and B3 are both $ø 1 \mathrm{~mm}$ balls placed in $ø 3 \mathrm{~mm}$ holes. Since the same gain $(41 \mathrm{~dB})$ has resulted in achieving black colour for these reflectors as well as the machining $ø 1.5 \mathrm{~mm} \mathrm{SDH}$, it can be concluded that $\mathrm{A} 3$ and $\mathrm{B} 3$ are $\geq \varnothing 1.5 \mathrm{~mm}$ (see Figure 8a,b and Figure 11). 
2) The assumption I (see Figure 9) is about the manufacturing process as there is uncertainty about the amount of $ø 3 \mathrm{~mm}$ hole filled by the filler wire during the deposition of the subsequent layer. There are two possibilities: (I1) the subsequent layer has quickly passed over the hole and then almost a $\varnothing 3 \mathrm{~mm}$ hole is remained or (I2) a random amount of the hole is filled with the filler wire. It is believed that the I2 cannot be true because both A3 and B3 appear in black with exactly the same gain (41 $\mathrm{dB}$ ) while if a random amount of air was available around the ball, this repeatability will be hard to be achieved. Furthermore, the manufacturing photographs don't show any major drop in the subsequent layer which was supposed to show a keyhole defect if the main part of the deposit had flowed inside the hole. It is then assumed that I1 is true and, therefore, the largest part of both A3 and B3 is $ø 3 \mathrm{~mm}$. If very low amplitude of $\varnothing 1 \mathrm{~mm}$ tungsten ball and a small melt droplet are ignored, this can be roughly considered as a ø3 $\mathrm{mm}$ spherical hole inside the WAAM sample which was impossible to be produced with any other manufacturing method. Therefore, a gain of $41 \mathrm{~dB}$ is required to detect a $ø 3 \mathrm{~mm}$ spherical hole in the WAAM sample (note this was $32 \mathrm{~dB}$ for a $ø 3 \mathrm{~mm} \mathrm{SDH}$ as shown in Figure 6).

3) D1, D2 and D3 are all ø3 $\mathrm{mm}$ tungsten carbide balls placed in $ø 3 \mathrm{~mm}$ hole and then no air around the ball is expected.

4) The conclusion 2 and 3 plus Figure 6 results are combined in the Chart 1 (see Figure 10 and Figure 11) which summarised the gain required to detect different $ø 3 \mathrm{~mm}$ defect types in both WAAM and reference sample (Figure $4 b$ ).

5) Comparison between $\varnothing 2 \mathrm{~mm}$ and $ø 3 \mathrm{~mm}$ balls shows that there is definitely some air around the $\varnothing 2 \mathrm{~mm}$ ball, otherwise, $\mathrm{C} 1-\mathrm{C} 3$ required $>12 \mathrm{~dB}$ gain increase while they are detected with just 6-7 $\mathrm{dB}$ (note the reflection amplitude from the air is higher than the tungsten ball).

6) $\mathrm{C} 3$ is a $ø 2 \mathrm{~mm}$ ball inside a $ø 3 \mathrm{~mm}$ hole but this cannot be a fully air-filled $ø 3 \mathrm{~mm}$ hole which needs just $41 \mathrm{~dB}$ to be detected, based on the results shown in Chart 1 , rather than $41+6 \mathrm{~dB}$ achieved for $\mathrm{C} 3$. Therefore, $\mathrm{C} 3$ is a combination of air and tungsten plus perhaps some steel moved inside the hole during the deposition.

7) The assumption II (see Figure 9) means that the ball is moved to the side of the hole, due to the shielding gas pressure, and then the difference between the hole diameter and the ball diameter $(0.5 \mathrm{~mm}$ for $\mathrm{C} 1$ and $\mathrm{C} 2$ but $1 \mathrm{~mm}$ for $\mathrm{C} 3)$ is considered as the dominant air reflector in these holes. Therefore, it is required to increase the gain to $47 \mathrm{~dB}$ for $ø 1 \mathrm{~mm}$ LoF (air around C3) and $48 \mathrm{~dB}$ for $ø 0.5 \mathrm{~mm} \mathrm{LoF}$ (air around C1-2) 
which results in the summary Chart 2 (see Figure 10). It is worth mentioning that any cavity (circular or linear) filled with air, rather than filler wire or embedded tungsten balls, is considered as LoF in this work.

8) $\mathrm{A} 1, \mathrm{~A} 2$ and B2 are all $ø 1 \mathrm{~mm}$ balls placed in $ø 1.5 \mathrm{~mm}$ hole and then, if Conclusion 7 was correct, these needed to be detected with $+7 \mathrm{~dB}$ same as $\mathrm{C} 1$ and $\mathrm{C} 2$ which both had $0.5 \mathrm{~mm}$ air around the tungsten ( $\varnothing 2 \mathrm{~mm}$ ball in $\varnothing 2.5 \mathrm{~mm}$ hole). This means that both assumption II and some part of the results in Chart 2 are incorrect.

9) Assumption III is considered in order to justify the problem discussed in conclusion 8 . This assumes that the small bottom part of the hole (produced due to the drill bit shape) is blocked by the tungsten ball and then hard to be filled with the melt. This is then $1.3 \mathrm{~mm}$ for a $ø 2 \mathrm{~mm}$ ball placed in a $ø 3 \mathrm{~mm}$ hole and so on, see Figure 9. Therefore, the gain required for $\mathrm{C} 1(\varnothing 2 \mathrm{~mm}$ ball in $\varnothing 2.5 \mathrm{~mm}$ hole and then $0.5 \mathrm{~mm}$ air or $\mathrm{LoF}$ ) which is $48 \mathrm{~dB}$ can be considered as the gain required for detection of $0.5 \mathrm{~mm}$ LoF in the WAAM sample. Similarly, the LoF size estimation (corrected version based on this new conclusion) can be summarised in Chart 3 (see Figure 10).

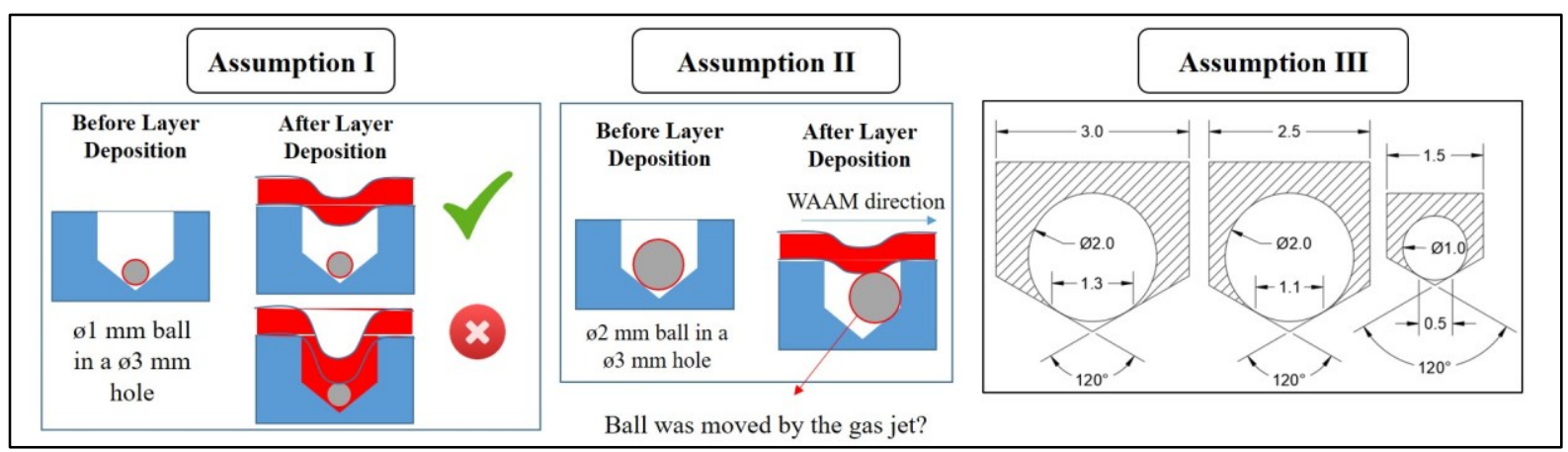

Figure 9. Manufacturing assumptions for the effect of deposition on the position of tungsten balls embedded in the WAAM sample

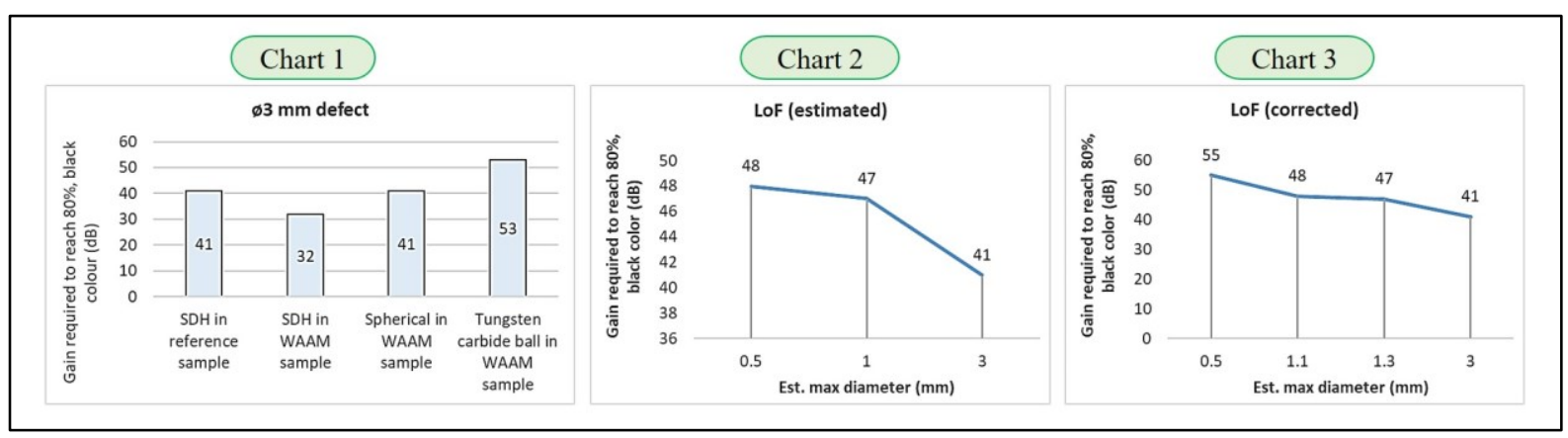

Figure 10. Summary charts to correlate the reflection gain with the defect (intentional defect or unknown size lack of fusion) size 


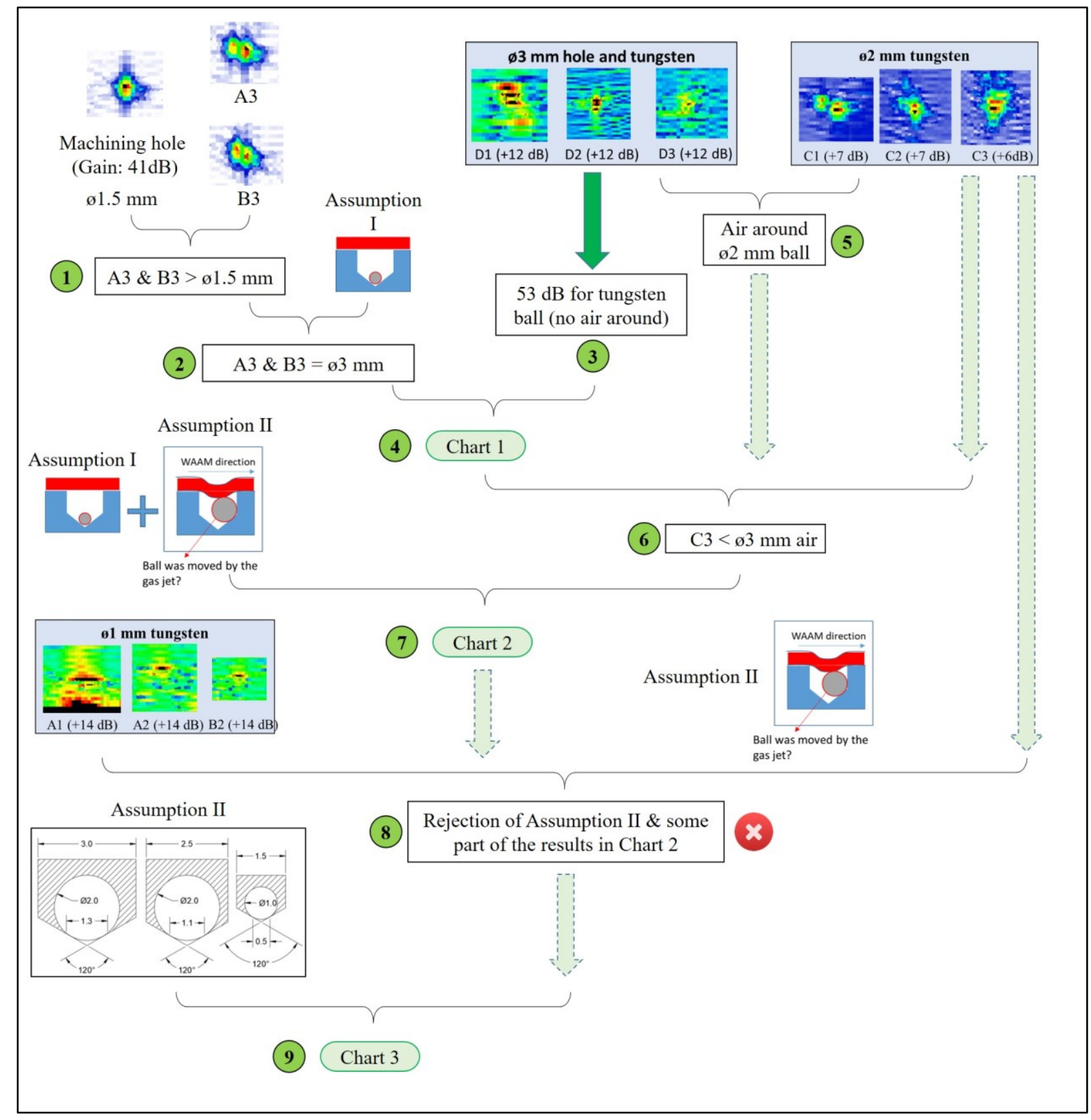

Figure 11. Conclusions of the step-by-step inspection and calibration procedure using tungsten embedded defects in the WAAM wall

Although the number of different size tungsten balls placed in different locations and inside different hole diameters resulted in a sophisticated setup, the achieved repeatability (see Figure 12) proves that the proposed calibration approach can be used to obtain consistent results and increase the robustness of ultrasonic inspection and defect sizing for WAAM samples. 


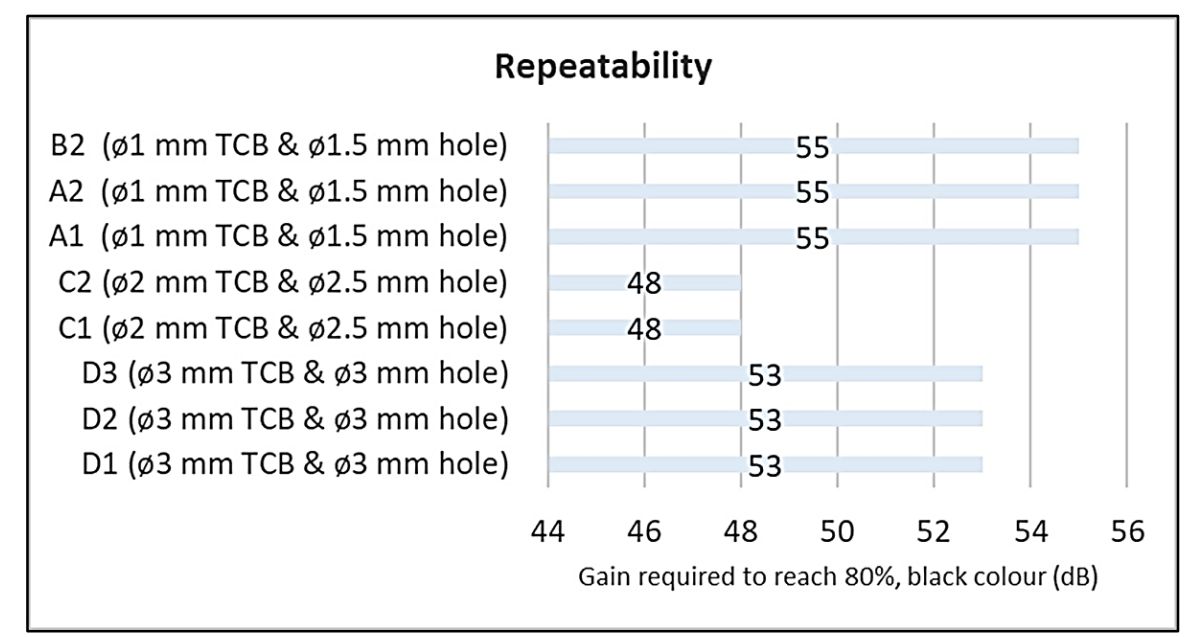

Figure 12. Repeatability achieved with the calibration procedure and intentional defects (TCB: tungsten carbide ball) used in this study

Chart 3 (Figure 10), developed in this study, is used for the prediction of the shape and size of an unknown LoF (see Figure 13). In this work, any cavity (circular or linear) filled with air, rather than metal, is called LoF. This is however different from the standard definition of linear LoF in the deposits but it has been chosen for ease of explanation since the determination of the defect type is excluded from this paper. The unknown LoF is then assumed to have a circular cross-section in YZ plane and its diameter is considered as the size of LoF. It is also known that Chart 3 has just four points and then linear interpolation is used to find the equivalent LoF size for the gains excluded from these points. The shape prediction and sizing are both estimated based on the calibration procedure and all discussion related to Figure 8. It was deemed critical to validate these estimations; therefore, the sample was slowly milled sidewise, with machining steps of $0.1 \mathrm{~mm}$, in order to monitor the appearance of the LoF and capture its largest extension on the XZ plane. After each machining step, a ruler was placed next to the LoF and then a photo was taken. The machining results (Figure 14a) and LoF measurements are compared with the phased array ultrasonic inspection in Figure 14b. This comparison shows the length and the maximum size of the LoF had been predicted accurately. This demonstrates that the ultrasonic phased array setup and methodology used in this study is able to detect the shape and estimate the size of unknown LoF in the WAAM sample. As no calibration standard for phased array inspection of the WAAM samples exists to date; the calibration procedure developed in this study, based on intentionally embedded tungsten carbide balls, has shown potential to be used for the defect sizing in steel WAAM components. 


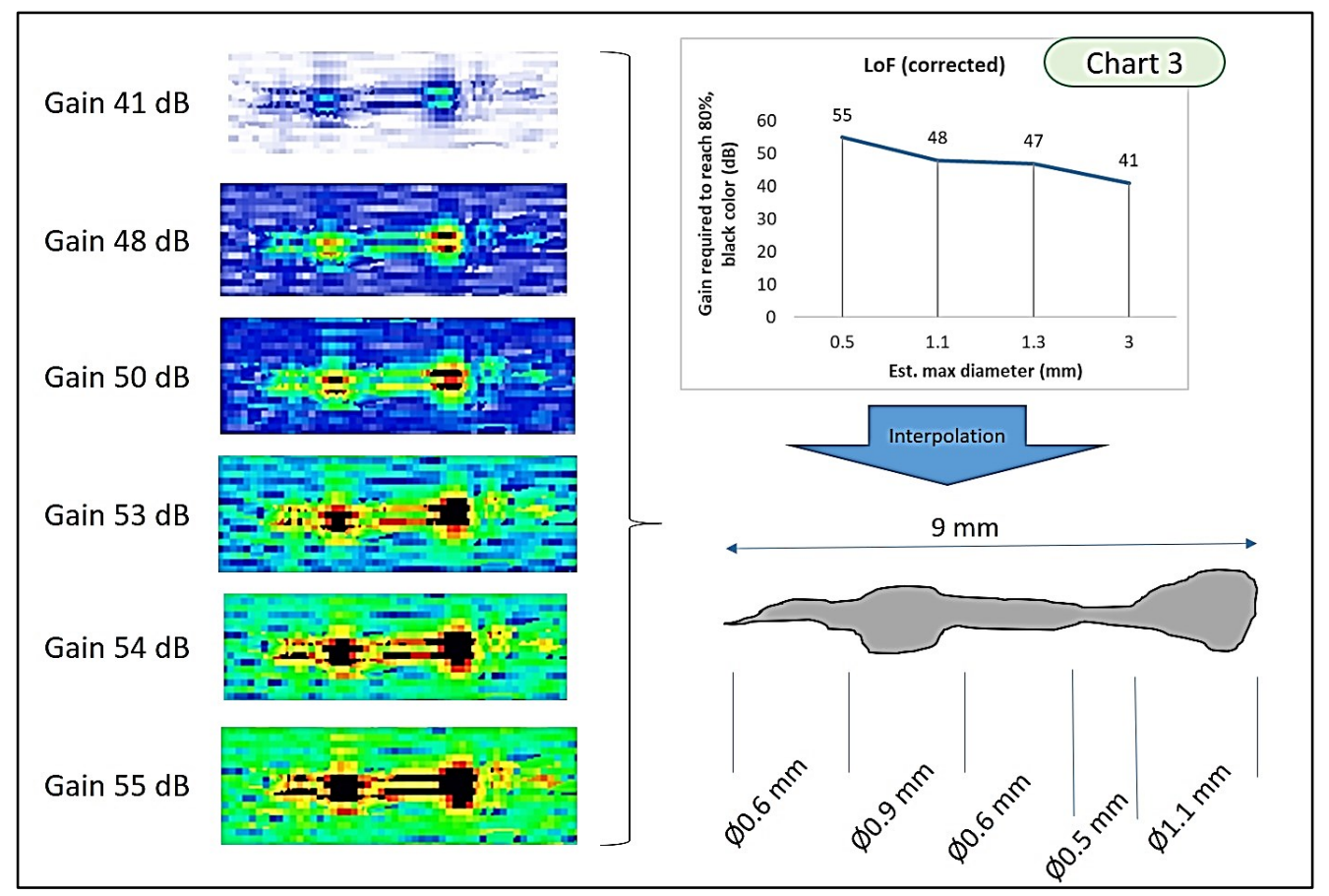

Figure 13. Sizing (estimated) of an unknown LoF using the calibration procedure developed in this study

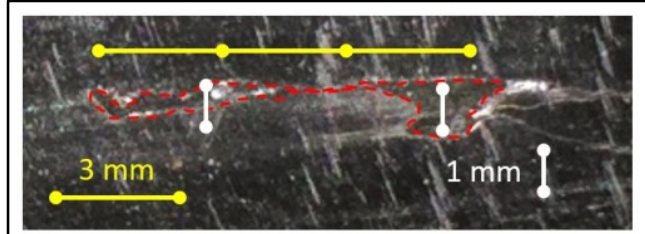

(a)

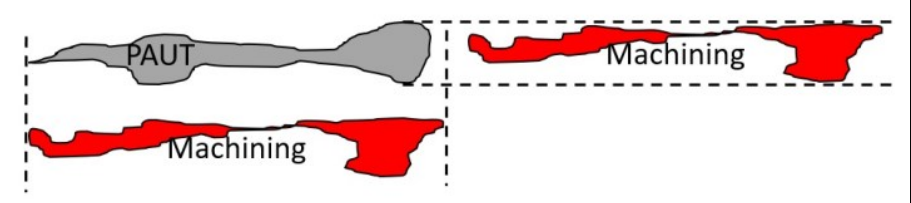

(b)

Figure 14. The LoF measurement after cutting the WAAM sample (a) and the same scale comparison of that with the phased array ultrasonic testing (PAUT) results (b)

\section{Conclusions}

In this study, a WAAM sample was manufactured with tungsten carbide balls intentionally embedded in the specimen. The sample was inspected using a TFM ultrasonic phased array approach. A calibration procedure was developed to enable accurate sizing of an unknown lack of fusion defect. Based on the achieved results, it can be concluded that:

1) TFM was able to detect most of the defects, however, a meandering scan is required to achieve the maximum possible amplitude for the reflectors.

2) The ultrasonic phased array system was first calibrated using SDH machined in the WAAM wall. 
3) Owing to the variation in the tungsten balls diameter, location and the drilled hole diameter, a step-by-step methodology is developed to achieve a chart for sizing of an unknown defect in the steel WAAM specimen.

4) Using the developed relationship between the defect size and the gain, shape and size of an unknown LoF was detected and a reasonable agreement was achieved when the inspection results were compared with the actual defect dimensions measured on the WAAM machined surface.

5) Because the methodology used here is validated, the assumptions made are considered as verified assumptions and then it can be claimed that a number of known size tungsten balls and known size near-spherical air-filled defects were successfully embedded in the WAAM wall using an intentional defect process. From a manufacturing point of view, embedding a known size air-filled defect is impractical with the other manufacturing methods. This shows the value of intentional defect process discussed here.

6) In comparison with the $ø 3 \mathrm{~mm}$ spherical air-filled defect, the $ø 3 \mathrm{~mm}$ tungsten carbide ball was detected with $+12 \mathrm{~dB}$ gain increase. This proves that the tungsten carbide balls, if they are successfully embedded during the WAAM process, are good reflectors of the ultrasonic wave and subsequent detection of them plus the controlled air-filled defects around them can also be beneficial for the NDT calibration and sizing purposes.

Finally, a comprehensive inspection strategy has been tested to detect and size steel WAAM defects. It is recommended to focus future work on the development of the same strategy for other WAAM materials (e.g. titanium) and for different additive manufacturing processes. Although CMT was used here, ultrasonic defect detection on samples produced with other methods (e.g. Laser metal deposition or plasma transferred arc WAAM) is worth studying in the future works.

\section{Acknowledgements}

This work was supported by an EPSRC project (EP/P031064/1) called Robotic Wire + Arc Additive Manufacture (RoboWAAM). The authors then like to acknowledge EPSRC for the support and funding of the project. 


\section{References}

[1] J. Coykendall, M. Cotteleer, J. Holdowsky, M. Mahto, 3D opportunity in aerospace and defense: additive manufacturing takes flight, A Deloitte series on additive manufacturing, Deloitte University Press, Westlake, TX, 2014.

[2] W.E. Frazier, Metal Additive Manufacturing: A Review, Journal of Materials Engineering and Performance 23 (2014) 1917-1928.

[3] U. Iftikhar, ADIRA TESTS SLM-XL LARGE SCALE 3D PRINTER PROTOTYPE WITH TECNICO LISBOA AND MANUEL CONCEIÇÃO GRAÇA, https://3dprintingindustry.com/, 2019.

[4] C. Vargel, Corrosion of Aluminium, Elsevier Ltd., Oxford, 2004.

[5] S.W. Williams, F. Martina, A.C. Addison, J. Ding, G. Pardal, P. Colegrove, Wire plus Arc Additive Manufacturing, Materials Science and Technology 32 (2016) 641-647.

[6] P. Colegrove, A. McAndrew, J. Ding, F. Martina, P. Kurzynski, S. Williams, System architectures for large scale wire + arc Additive Manufacture, 10th International Conference on Trends in Welding Research, Japan Welding Society, Hitotsubashi Hall, Tokyo, JAPAN, 2016.

[7] C. University, Is this the largest metal 3D part ever made?, Cranfield University, UK, 2016.

[8] D.H. Ding, Z.X. Pan, D. Cuiuri, H.J. Li, Wire-feed additive manufacturing of metal components: technologies, developments and future interests, International Journal of Advanced Manufacturing Technology 81 (2015) 465-481.

[9] F.D. Wang, S. Williams, P. Colegrove, A.A. Antonysamy, Microstructure and Mechanical Properties of Wire and Arc Additive Manufactured Ti-6Al-4V, Metallurgical and Materials Transactions a-Physical Metallurgy and Materials Science 44A (2013) 968-977. [10] C.N. Macleod, G. Dobie, S.G. Pierce, R. Summan, M. Morozov, Machining-Based Coverage Path Planning for Automated Structural Inspection, Ieee Transactions on Automation Science and Engineering 15 (2018) 202-213.

[11] G. Dobie, R. Summan, S.G. Pierce, W. Galbraith, G. Hayward, A Noncontact Ultrasonic Platform for Structural Inspection, Ieee Sensors Journal 11 (2011) 2458-2468.

[12] R.S. Lim, H.M. La, W.H. Sheng, A Robotic Crack Inspection and Mapping System for Bridge Deck Maintenance, Ieee Transactions on Automation Science and Engineering 11 (2014) 367-378.

[13] P.A. Hancock, M.H. Chignell, MENTAL WORKLOAD DYNAMICS IN ADAPTIVE INTERFACE DESIGN, Ieee Transactions on Systems Man and Cybernetics 18 (1988) 647658.

[14] P. Cawley, Non-destructive testing - current capabilities and future directions, Proc. Inst. Mech. Eng. Pt. L-J. Mater.-Design Appl. 215 (2001) 213-223.

[15] B.W. Drinkwater, P.D. Wilcox, Ultrasonic arrays for non-destructive evaluation: A review, Ndt \& E International 39 (2006) 525-541.

[16] C. Holmes, B.W. Drinkwater, P.D. Wilcox, Advanced post-processing for scanned ultrasonic arrays: Application to defect detection and classification in non-destructive evaluation, Ultrasonics 48 (2008) 636-642.

[17] C. Holmes, B.W. Drinkwater, P.D. Wilcox, Post-processing of the full matrix of ultrasonic transmit-receive array data for non-destructive evaluation, Ndt \& E International 38 (2005) 701-711.

[18] Y. Mendelsohn, E. Wiener-Avnear, Simulations of circular 2D phase-array ultrasonic imaging transducers, Ultrasonics 39 (2002) 657-666. 
[19] K.J. Kirk, A. McNab, A. Cochran, I. Hall, G. Hayward, Ultrasonic arrays for monitoring cracks in an industrial plant at high temperatures, Ieee Transactions on Ultrasonics Ferroelectrics and Frequency Control 46 (1999) 311-319.

[20] S. Chatillon, G. Cattiaux, M. Serre, O. Roy, Ultrasonic non-destructive testing of pieces of complex geometry with a flexible phased array transducer, Ultrasonics 38 (2000) 131-134. [21] A. Neild, D.A. Hutchins, T.J. Robertson, L.A.J. Davis, D.R. Billson, The radiated fields of focussing air-coupled ultrasonic phased arrays, Ultrasonics 43 (2005) 183-195.

[22] A. Gachagan, S. Kelly, M. Altman, E. Grauvogl, G. Hayward, R. Banks, T. McCunnie, D. Skillen, Development of a manual air-coupled ultrasonic inspection instrument for use on aeronautical structures under in-service conditions, Review of Progress in Quantitative Nondestructive Evaluation, Vols 22a and 22b 20 (2003) 883-890.

[23] A. Lopez, R. Bacelar, I. Pires, T.G. Santos, J.P. Sousa, L. Quintino, Non-destructive testing application of radiography and ultrasound for wire and arc additive manufacturing, Additive Manufacturing 21 (2018) 298-306.

[24] C.-P. Bazin de Caix, A. Gigant, ISO/TC 261: Additive manufacturing, in: B. Dutton (Ed.) NDT for AM parts, International Organization for Standardization, 2018.

[25] D. Clark, S.D. Sharples, D.C. Wright, Development of online inspection for additive manufacturing products, Insight 53 (2011) 610-613.

[26] A. Lopez, R. Bacelar, I. Pires, T. Santos, L. Quintino, MAPPING OF NONDESTRUCTIVE TECHNIQUES FOR INSPECTION OF WIRE AND ARC ADDITIVE MANUFACTURING, Proceedings of the 7th International Conference on Mechanics and Materials in Design (M2d2017) (2017) 1829-1844.

[27] Y. Javadi, C.N. MacLeod, S.G. Pierce, A. Gachagan, W. Kerr, J. Ding, S. Williams, M. Vasilev, R. Su, C. Mineo, J. Dziewierz, Ultrasonic phased array inspection of wire plus arc additive manufacture (WAAM) samples using conventional and total focusing method (TFM) imaging approaches, Insight 61 (2019) 144-148.

[28] M. Consonni, C.F. Wee, C. Schneider, Manufacturing of welded joints with realistic defects, Insight 54 (2012) 76-+.

[29] Y. Javadi, M. Vasilev, C.N. MacLeod, S.G. Pierce, R. Su, C. Mineo, J. Dziewierz, A. Gachagan, Intentional weld defect process: From manufacturing by robotic welding machine to inspection using TFM phased array, AIP Conference Proceedings 2102 (2019) 040011. [30] R. Biswal, X. Zhang, A.K. Syed, M. Awd, J. Ding, F. Walther, S. Williams, Criticality of porosity defects on the fatigue performance of wire + arc additive manufactured titanium alloy, International Journal of Fatigue 122 (2019) 208-217.

[31] J. Gu, J. Ding, S.W. Williams, H. Gu, P. Ma, Y. Zhai, The effect of inter-layer cold working and post-deposition heat treatment on porosity in additively manufactured aluminum alloys, Journal of Materials Processing Technology 230 (2016) 26-34. 